\title{
Minimizing crack energy release rate by topology optimization
}

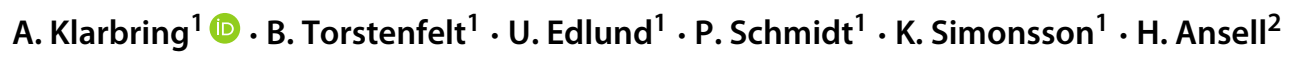 \\ Received: 25 September 2017 / Revised: 10 April 2018 / Accepted: 11 April 2018 / Published online: 26 April 2018 \\ (c) The Author(s) 2018
}

\begin{abstract}
Fatigue cracked primary aircraft structural parts that cannot be replaced need to be repaired by other means. A structurally efficient repair method is to use adhesively bonded patches as reinforcements. This paper considers optimal design of such patches by minimizing the crack extension energy release rate. A new topology optimization method using this objective is developed as an extension of the standard SIMP compliance optimization method. The method is applied to a cracked test specimen that resembles what could be found in a real fuselage and the results show that an optimized adhesively bonded repair patch effectively reduces the crack energy release rate.
\end{abstract}

Keywords Topology optimization · SIMP · Crack energy release rate $\cdot$ Virtual crack extension method

\section{Introduction}

The development of the topology optimization method described in this paper is driven by a clear practical application, namely the repair of aircraft parts containing cracks. Primary load carrying structures in fuselages are mainly manufactured from machined and/or forged single piece parts. Such parts are weight optimized and contain most often stress raisers of various kinds, e.g., cut-outs, thickness steps and interacting radii which make them prone to fatigue failure. The number of such hot-spots seems to increase when designing using digital modeling techniques, thus pushing the failure probability to rise and strain the risk budget. If fatigue cracking occurs during service life it is costly and impractical or most often impossible to replace these parts. The complexity of a fuselage structure is seen in Fig. 1. Fatigue cracked primary aircraft structural parts that cannot be replaced need to be repaired by other means. A structurally efficient such repair method is to use adhesively bonded patches as reinforcements. These

\footnotetext{
Responsible Editor: Ole Sigmund

$\triangle$ A. Klarbring

anders.klarbring@liu.se

1 Solid Mechanics, Department of Management and Engineering, Institute of Technology, Linköping University, 58183 Linköping, Sweden

2 Saab Aeronautics, 58254 Linköping, Sweden
}

patches should then be shaped so that the material is used as efficiently as possible. Therefore, we have developed a topology optimization method where the crack energy release rate is minimized. Such an objective, as explained below, is most natural since it should minimize and possibly eliminate the risk for fatigue failure by further growth of the crack. As far as we know this is the first time the crack energy release rate is used as an objective function in topology optimization. The method developed and demonstrated is a direct extension of a classical treatment of compliance topology optimization (Bendsøe and Sigmund 2002; Christensen and Klarbring 2009), which is a formulation used also for comparison in the test examples. This means that we use a SIMP penalization (Solid Isotropic Material with Penalization) (Rozvany et al. 1992) and regularize the problem by using Sigmund's sensitivity filtering (Sigmund 1994). The standard optimality criteria update formula (Bendsøe and Sigmund 2002; Christensen and Klarbring 2009) is slightly modified since the sensitivity of crack energy release rate, derived in the paper, is not restricted in sign.

For the aero structural applications motivating the developments in this paper, except for very short cracks, small scale yielding conditions can be assumed to prevail, meaning that inelastic deformations are restricted to a small regime at the crack front, and that the behavior of the crack is governed by the elastic stress state in the material surrounding this plastic zone, see e.g. Anderson (2017). In such a linear elastic fracture mechanics context, the intensity of the loading as seen from the crack can be described 


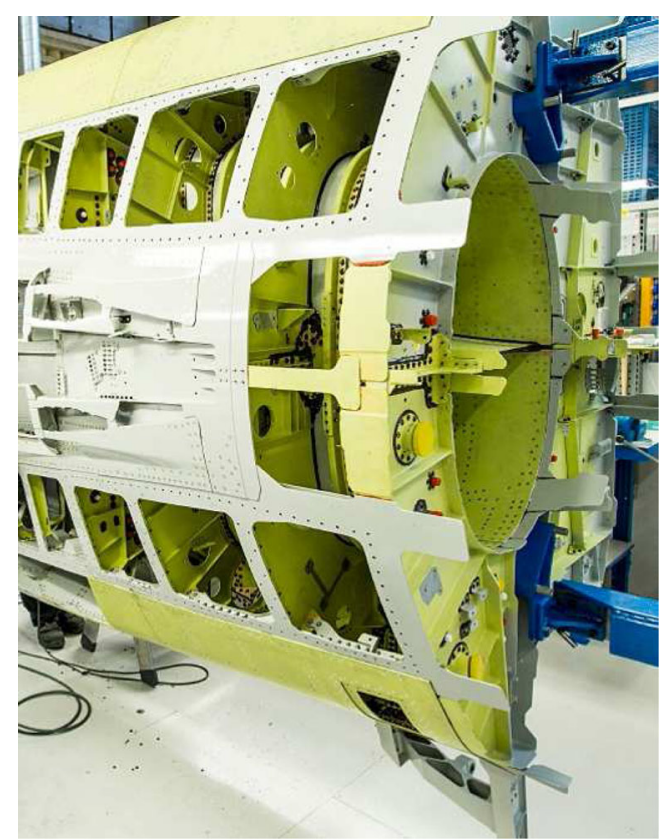

Fig. 1 The complexity of a fuselage structure makes it impractical and sometimes impossible to replace cracked parts. The application of adhesively bounded repair patches then becomes a useful repair method

by the stress intensity factors $K_{I}, K_{I I}$ and $K_{I I I}$, which for a plane geometry characterizes the stress singularity in tearing, in-plane shearing and out-of-plane shearing. For a general geometry and loading, however, a more direct description of the crack driving force is the energy release rate $G$, specifying the energy available for crack growth. In terms of equilibrium potential energy $\Pi$, seen as function of a crack area $A$, we may write $G=-\partial \Pi / \partial A$. For a plane geometry and for an isotropic material the stress intensity factors and $G$ are related as

$G=\frac{K_{I}^{2}}{E}+\frac{K_{I I}^{2}}{E}+\frac{K_{I I I}^{2}}{2 \mu}$

where $E$ is Young's modulus in case of plane stress or a modification valid for plane strain, and $\mu$ is the shear modulus. In a three-dimensional context, $G$ needs to be evaluated locally along the crack front (Anderson 2017). For a given load history the maximum value of $G$ governs static fracture, and its range governs fatigue crack propagation. Thus, there is a huge interest in finding ways to lower $G$ for cracks formed in primary aircraft structures, which is the topic of this work.

The paper is organized as follows: Section 2 introduces the finite element discretized structural problem. We use the basic definition of crack energy release rate as the sensitivity of the potential energy with respect to crack extension, and derive its sensitivity with respect to design changes. Such first and second variations of energy with respect to change of domain due to crack extension, and its relation to, e.g., the $J$-integral, is discussed in Nguyen (2000). In Section 3 the general structural optimization problem is introduced. Basic facts concerning SIMP penalization, filter regularization and the optimality criteria method are given. Calculation of the crack energy release rate as well as its sensitivity requires the sensitivity of the stiffness matrix with respect to crack extension. This is accomplished by the so-called virtual crack extension method (Parks 1974; Hellen 1975). In Section 4 we present numerical examples corresponding to two test specimen geometries and loadings. We present optimal repair patch geometries created by both an essentially standard compliance optimization formulation and the novel formulation that uses the crack energy release rate as objective. The more advanced specimen is chosen to resemble conditions that can be expected in a real fuselage. The model includes an adhesive layers that bonds the repair patches to the cracked specimen. Finally, summary and conclusions are given in Section 5.

\section{State problem, energy release rate and sensitivity}

Anticipating a finite element discretization of the cracked structure, we treat the problem in a discrete setting (Fig. 2). The following variables represent the design and state of the structure:

- The crack geometry is described by variables $\ell_{i}, i=$ $1, \ldots, n_{c}$, normalized to have the physical dimension
Fig. 2 Left: A structure containing a crack of length $\ell$. A schematic finite element discretization and corresponding nodal forces $\boldsymbol{F}$ and displacements $\boldsymbol{u}$ are shown. Right: A repair patch has been attached in order to mitigate the risk for further crack growth
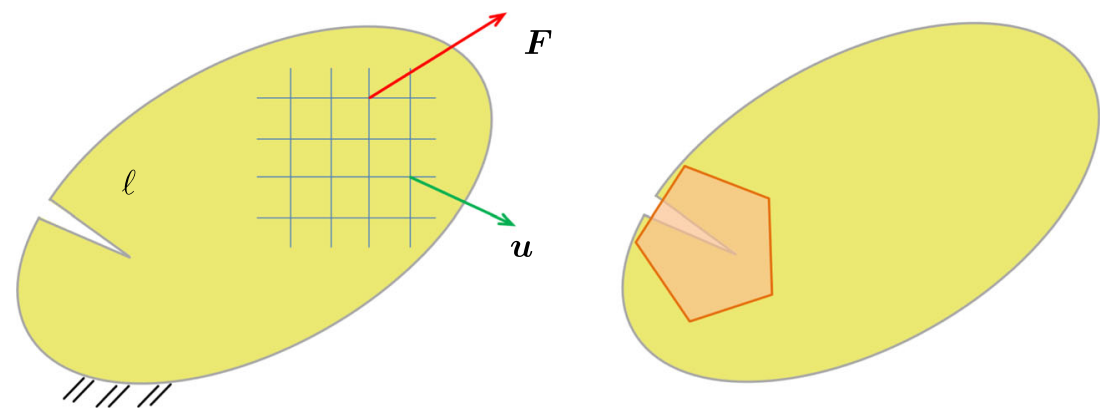
of surface area. For a two-dimensional problem, $\ell_{i}$ represents the length of crack $i$, multiplied by a constant thickness. In the three-dimensional case, these variables represent a parametrization of the crack shape, e.g., positions of finite element nodes along the crack front. The particular choice of such a parametrization used in the numerical examples will be discussed in detail in Section 3, but it may be noted that we use a linear interpolation of the crack front even though higher order displacement elements are used. Note also that all examples are modeled using three-dimensional geometry.

- The standard density-like variables of topology optimization, taking values in the closed interval between 0 and 1 , are $\rho_{i}, i=1, \ldots, n_{d}$ (Bendsøe and Sigmund 2002; Christensen and Klarbring 2009). In a two-dimensional problem they may be interpreted as thicknesses of the structural domain, while in three dimensions they can be seen as generalized densities, where the value 1 represents presence of material and 0 represents void.

- Displacements of finite element nodes are gathered in a vector $\boldsymbol{u}$, and $\boldsymbol{F}$ represents corresponding nodal force values.

The potential energy of the structure is defined as follows:

$\Pi(\boldsymbol{u}, \boldsymbol{\rho}, \ell)=\frac{1}{2} \boldsymbol{u}^{T} \boldsymbol{K}(\boldsymbol{\rho}, \ell) \boldsymbol{u}-\boldsymbol{F}^{T} \boldsymbol{u}$,

where $\rho=\left(\rho_{1}, \ldots, \rho_{n_{d}}\right)^{T}, \ell=\left(\ell_{1}, \ldots, \ell_{n_{c}}\right)^{T}$, and where $K(\rho, \ell)$ is the stiffness matrix. It is assumed that the structure is sufficiently anchored by prescribed displacements so that this matrix is positive definite for any $\ell$ and $\rho_{i} \geq \varepsilon$, where $\varepsilon>0$ is an arbitrary small number. For simplicity, and in accordance with our example, we assume that the external given force $\boldsymbol{F}$ is independent of the design $\boldsymbol{\rho}$, which precludes, e.g., gravitational forces or non-zero prescribed displacements.

The equilibrium equation is given as a stationary point of the potential energy, i.e.,

$\frac{\partial \Pi(\boldsymbol{u}, \boldsymbol{\rho}, \ell)}{\partial \boldsymbol{u}}=\boldsymbol{K}(\boldsymbol{\rho}, \ell) \boldsymbol{u}-\boldsymbol{F}=\mathbf{0}$,

For $\rho_{i} \geq \varepsilon$, i.e., for a nonsingular $\boldsymbol{K}(\boldsymbol{\rho}, \ell)$, we can solve this equation to obtain the displacement as a function of the design and the crack geometry, i.e.,

$\boldsymbol{u}=\boldsymbol{u}(\rho, \ell)$

An energy release rate for a crack extension is associated to each crack parameter $\ell_{i}$ as

$G_{i}(\rho, \ell)=-\frac{d}{d \ell_{i}} \Pi(\boldsymbol{u}(\rho, \ell), \rho, \ell)$.
By direct differentiation, omitting arguments for $\boldsymbol{u}(\boldsymbol{\rho}, \ell)$, we find

$$
\begin{aligned}
\frac{d}{d \ell_{i}} \Pi(\boldsymbol{u}(\boldsymbol{\rho}, \ell), \boldsymbol{\rho}, \ell)= & \frac{1}{2}\left(\frac{\partial \boldsymbol{u}}{\partial \ell_{i}}\right)^{T} \boldsymbol{K}(\boldsymbol{\rho}, \ell) \boldsymbol{u}+\frac{1}{2} \boldsymbol{u}^{T} \boldsymbol{K}(\boldsymbol{\rho}, \ell) \frac{\partial \boldsymbol{u}}{\partial \ell_{i}} \\
& +\frac{1}{2} \boldsymbol{u}^{T} \frac{\partial \boldsymbol{K}(\boldsymbol{\rho}, \ell)}{\partial \ell_{i}} \boldsymbol{u}-\boldsymbol{F}^{T} \frac{\partial \boldsymbol{u}}{\partial \ell_{i}}
\end{aligned}
$$

Using the symmetry of $\boldsymbol{K}(\boldsymbol{\rho}, \ell)$ and the equilibrium (1) we obtain

$G_{i}(\rho, \ell)=-\frac{1}{2} \boldsymbol{u}(\rho, \ell)^{T} \frac{\partial \boldsymbol{K}(\rho, \ell)}{\partial \ell_{i}} \boldsymbol{u}(\rho, \ell)$.

Although this equation is calculated for zero-valued prescribed displacements, it turns out to be valid also for non-zero prescribed displacements (Klarbring and Strömberg 2012) (known as displacement control in fracture mechanics).

In the following we will also need the sensitivity of $G_{i}$ with respect to the design $\rho$, i.e., the mixed second derivative of $\Pi(\boldsymbol{u}(\boldsymbol{\rho}, \ell), \boldsymbol{\rho}, \ell)$ with respect to $\ell_{i}$ and $\rho_{j}$. Assuming continuity, the differentiation can be done in any order. A first differentiation of $\Pi$ with respect to $\rho_{i}$ gives the same expression as on the right hand side of (3), except for a replacement of $\ell_{i}$ by $\rho_{i}$. Differentiating this result with respect to $\ell_{i}$ gives

$$
\begin{array}{r}
\frac{d}{d \ell_{i}} \frac{d}{d \rho_{j}} \Pi(\boldsymbol{u}(\boldsymbol{\rho}, \ell), \boldsymbol{\rho}, \ell)=\frac{1}{2}\left(\frac{\partial \boldsymbol{u}}{\partial \ell_{i}}\right)^{T} \frac{\partial \boldsymbol{K}(\boldsymbol{\rho}, \ell)}{\partial \rho_{j}} \boldsymbol{u} \\
+\frac{1}{2} \boldsymbol{u}^{T} \frac{\partial \boldsymbol{K}(\boldsymbol{\rho}, \ell)}{\partial \rho_{j}} \frac{\partial \boldsymbol{u}}{\partial \ell_{i}}+\frac{1}{2} \boldsymbol{u}^{T} \frac{\partial^{2} \boldsymbol{K}(\boldsymbol{\rho}, \boldsymbol{\ell})}{\partial \rho_{j} \partial \ell_{i}} \boldsymbol{u} .
\end{array}
$$

From the symmetry of $\partial \boldsymbol{K}(\boldsymbol{\rho}, \ell) / \partial \rho_{j}$ we thus obtain

$\frac{\partial}{\partial \rho_{j}} G_{i}(\boldsymbol{\rho}, \ell)=\boldsymbol{u}^{T} \frac{\partial \boldsymbol{K}(\boldsymbol{\rho}, \ell)}{\partial \rho_{j}} \lambda_{i}-\frac{1}{2} \boldsymbol{u}^{T} \frac{\partial^{2} \boldsymbol{K}(\boldsymbol{\rho}, \ell)}{\partial \rho_{j} \partial \ell_{i}} \boldsymbol{u}$,

where, from (1), $\lambda_{i}=-\partial \boldsymbol{u} / \partial \ell_{i}$ is the solution of

$\boldsymbol{K}(\boldsymbol{\rho}, \ell) \lambda_{i}=\frac{\partial \boldsymbol{K}(\boldsymbol{\rho}, \ell)}{\partial \ell_{i}} \boldsymbol{u}$,

where $\boldsymbol{u}$ solves (1). Thus, calculation of the $G_{i}:$ s and their design sensitivities hinges on calculating derivatives of the stiffness matrix. This will be discussed in the next section after introducing a more precise form of $\boldsymbol{K}(\rho, \ell)$.

\section{Optimization problems and solution algorithm}

Given a goal or objective function $f(\boldsymbol{\rho})$, a general form of the optimization problem reads

$$
\left\{\begin{array}{l}
\min _{\boldsymbol{\rho}} f(\boldsymbol{\rho}) \\
\text { subject to }\left\{\begin{array}{l}
\sum_{i=1}^{n_{d}} \rho_{i} v_{i}=V \\
\varepsilon \leq \rho_{i} \leq 1, i=1, \ldots, n_{d},
\end{array}\right.
\end{array}\right.
$$


where $V$ is the amount of volume of material to be used in the design and $v_{i}$ is the volume associated to a value of $\rho_{i}=$ 1 of design variable $i$. If there is at most one design variable for each finite element, as in the numerical examples in Section $4, v_{i}$ represents the total volume of finite element $i$. Note that the reversed version of this optimization problem is certainly a possible formulation, i.e., minimizing mass (or volume) under constraints on the function $f$. When the objective function $f$ represents strain energy release rate, which is the prime case in this paper, this would mean securing that $G_{i}$ stays below a critical value. However, from a practical point of view the above formulation achieves this result by solving for a sequence of volumes, essentially producing a Pareto front for a multi-objective problem.

We will consider two different objective functions $f$ in the following. Firstly, mainly for comparison, we consider the standard compliance objective:

$f_{c}(\boldsymbol{\rho})=\frac{1}{2} \boldsymbol{F}^{T} \boldsymbol{u}(\boldsymbol{\rho}, \boldsymbol{\ell})$,

where the crack geometry, i.e., $\ell$, is considered fixed.

Secondly, we consider the minimization of the energy release rate for a certain advancement of the crack. This means choosing how to aggregate the $n_{c}$ release rates $G_{i}$ into one scalar function. In this work we simply use a summation, i.e., the objective function is

$f_{g}(\boldsymbol{\rho})=\sum_{i=1}^{n_{c}} G_{i}(\boldsymbol{\rho}, \boldsymbol{\ell})$.

For a sheet-like structure modeled in three dimensions and having one through-thickness crack, where $\ell_{i}$ represents positions of finite element nodes along the crack front, such an $f_{g}(\rho)$ represents the energy release rate for an advancement of the crack keeping the crack front straight. This is applicable to the example in Section 4, but other ways of forming a scalar function could be considered in other situations. For instance, one could use the maximum of all $G_{i}: \mathrm{s}$, or a $p$-norm approximation of such a maximum function, which is well known in other topology optimization applications (Holmberg et al. 2013).

At least for the three-dimensional case there is no clear physical interpretation of designs $\rho_{i}$ strictly inside the interval $\varepsilon \leq \rho_{i} \leq 1$, and therefore the optimal design should preferably be such that $\rho_{i}=\varepsilon \approx 0$, indicating no material, or $\rho_{i}=1$, indicating material. The standard procedure for achieving this, in case of the compliance objective $f_{c}$, is to use penalization. In the so-called SIMP method such penalization follows by forming the stiffness matrix as

$\boldsymbol{K}(\boldsymbol{\rho}, \boldsymbol{\ell})=\sum_{i=1}^{n_{d}} \rho_{i}^{q} \boldsymbol{K}_{i}(\boldsymbol{\ell})+\hat{\boldsymbol{K}}(\boldsymbol{\ell})$,

where the first term corresponds to the part of the structure that is subject to design and the second term represents the stiffness of the rest of the structure. Assuming one design variable for each finite element of the design part, $n_{d}$ is the number of such elements and $\boldsymbol{K}_{i}(\boldsymbol{\ell})$ is the global element stiffness matrix for element $i$ and for a unit value of the corresponding design variable $\rho_{i}$. The parameter $q>1$ is a penalty parameter usually given the value 3 (Bendsøe and Sigmund 2002). In this work we apply such a SIMP penalization also when using $f_{g}$ as objective. It turns out that also in this case the result is a near 0-1 design.

Looking now at (3), (5) and (6), we note that calculation of $G_{i}$ and its sensitivity requires derivatives of the stiffness matrix given in (7). The first derivative is

$\frac{\partial \boldsymbol{K}(\boldsymbol{\rho}, \boldsymbol{\ell})}{\partial \rho_{i}}=q \rho_{i}^{q-1} \boldsymbol{K}_{i}(\boldsymbol{\ell})$,

and the second derivative of $\boldsymbol{K}(\boldsymbol{\rho}, \boldsymbol{\ell})$, in the second term of (5), is zero if all $\boldsymbol{K}_{i}(\boldsymbol{\ell})$ :s are independent of $\boldsymbol{\ell}$. This is so if the design part of the structure does not contain crack fronts, which is the case in our application to repair patches. The derivative $\partial \boldsymbol{K}(\boldsymbol{\rho}, \ell) / \partial \ell_{i}$, needed to calculate the $G_{i}$ :s and their derivatives, is obtained by the virtual crack extension method (Parks 1974; Hellen 1975). There is by now a large body of literature associated with this method, but from a structural optimization point of view it is interesting to see the analogy to discrete shape sensitivity (Christensen and Klarbring 2009). The calculation of the stiffness matrix derivative can be performed numerically by a finite difference expression, as was originally proposed in Parks (1974) and Hellen (1975) (semi-analytical sensitivity analysis), or by analytical expressions (Giner et al. 2002; Waisman 2010), similar to those for shape sensitivity of isoparametric finite elements first derived in Brockman (1987). In the numerical examples we have used a semianalytical approach. The vicinity of the crack is modeled by parabolic elements, meaning that the crack front is interpolated as piecewise second order curves. However, crack shape variables $\ell_{i}$ are associated only to corner nodes, implying a piecewise linear interpolation where an incremental change of $\ell_{i}$ is associated to creation of a small surface element that is triangular for a straight crack front. This method is remarkably stable with respect to step length $\Delta \ell_{i}$ of the finite difference expression. As a default value $\Delta \ell_{i}$ corresponds to a movement of the node $10^{-4}$ times the element length, but numerical tests show that the result remains stable for values from $10^{-2}$ to $10^{-5}$ (Johansson 1996). Concerning sensitivity of the results with respect to spatial mesh refinement this is investigated in our first example of Section 4.

The sensitivity of $f_{c}$ follows from a derivation similar to the derivation of the expression (3) for the energy release 
rate $G_{i}$. Using the fact that $f_{c}(\boldsymbol{\rho})=-\Pi(\boldsymbol{u}(\boldsymbol{\rho}, \ell), \boldsymbol{\rho}, \ell)$ one obtains

$\frac{\partial f_{c}(\boldsymbol{\rho})}{\partial \rho_{i}}=-\frac{1}{2} \boldsymbol{u}(\boldsymbol{\rho}, \ell)^{T} \frac{\partial \boldsymbol{K}(\boldsymbol{\rho}, \ell)}{\partial \rho_{i}} \boldsymbol{u}(\boldsymbol{\rho}, \ell)$.

Analogous to (3), this expression for the sensitivity is valid also for non-zero prescribed displacements (Klarbring and Strömberg 2012).

The introduction of a SIMP penalization parameter $q$ different from 1 , for the compliance objective $f_{c}$, usually results in anomalies labeled mesh-dependency and checkerboarding (Bendsøe and Sigmund 2002; Christensen and Klarbring 2009; Sigmund and Petersson 1998). The mostly used remedy for this is filtering which could be applied directly to the design variables or to the sensitives. The latter was suggested by Sigmund (1994) and is used in this work for both $f_{c}$ and $f_{g}$. In the following a filtered sensitivity is denoted by a hat symbol. However, since nonpositiveness of (5) cannot be guaranteed, which it can for (8), we use different modified sensitivities depending on the objective function. Such sensitives $S\left(\rho^{k}\right)$ at the current design $\rho^{k}$ are:

$S\left(\boldsymbol{\rho}^{k}\right)=\frac{\partial \widehat{f_{c}\left(\boldsymbol{\rho}^{k}\right)}}{\partial \rho_{i}} \quad$ or $S\left(\boldsymbol{\rho}^{k}\right)=\min \left[0, \frac{\partial \widehat{f_{g}\left(\boldsymbol{\rho}^{k}\right)}}{\partial \rho_{i}}\right]$,

where, as indicated above, a hat denotes Sigmund sensitivity filtering.

The optimality criteria iteration now starts by an initial calculation of trial designs for a current Lagrangian multiplier $\Lambda$ :

$\hat{\rho}_{i}^{k+1}(\Lambda)=\rho_{i}^{k}\left[\frac{-S\left(\rho^{k}\right)}{v_{i} \Lambda}\right]^{\eta}$,

where $\eta<1$ is a positive damping coefficient This trial design is then modified according to the box constraint of $(\mathbb{P})$ :

$\rho_{i}^{k+1}(\Lambda)= \begin{cases}\varepsilon & \text { if } \hat{\rho}_{i}^{k+1}(\Lambda)<\varepsilon \\ \hat{\rho}_{i}^{k+1}(\Lambda) & \text { if } \varepsilon \leq \hat{\rho}_{i}^{k+1}(\Lambda) \leq 1 \\ 1 & \text { if } \hat{\rho}_{i}^{k+1}(\Lambda)>1 .\end{cases}$
Finally, $\Lambda$ needs to be adjusted so that

$\sum_{i=1}^{n_{d}} \rho_{i}^{k+1}(\Lambda) v_{i}=V$

This may be accomplished by simple interval reduction. The algorithm then returns to calculate a new trial design. Convergence is assumed when the maximum absolute difference between $\rho_{i}^{k}$ and $\rho_{i}^{k+1}$ falls below a prescribed value, or after a fixed number of iterations. The need for a non-positive $S\left(\rho^{k}\right)$ follows from the iteration formula (9) if the Lagrangian multiplier is assumed positive: a fact that can be expected as long as a larger available volume $V$ implies a lower optimal objective function value.

The method has been implemented in the in-house FE program Trinitas (The TRINITAS Project 2017).

\section{Examples}

We consider two test problems based on the specimens shown in Figs. 3 and 7. In the first problem the loading and modeling of the repair patches are chosen to be as simple as possible, eliminating any bending action, while in the second problem we intend to resemble a geometry and loading that could be present in a real fuselage. The material is the same in both problems. Both the cracked structures and the repair patches consist of aluminum having a Young's modulus of $71000 \mathrm{MPa}$ and a Poisson's ratio of 0.33 . The repair patches are fitted to the cracked structures by adhesive layers of thickness $0.2 \mathrm{~mm}$ that is modeled as a linear elastic isotropic continuum with a shear modulus of $860 \mathrm{MPa}$ and a Poisson's ratio of 0.38 . Concerning the modeling of the part of the adhesive layer that is on top of a crack, there are two distinct possibilities: either the layer is taken as intact or as broken according to the crack geometry. We have chosen the latter since this is in line with experimental findings. However, since the adhesive material is weak compared to that of aluminum this choice should not have a large impact on the result.
Fig. 3 A test specimen with a crack shown as a read color line. The distributed loading is shown as green arrows

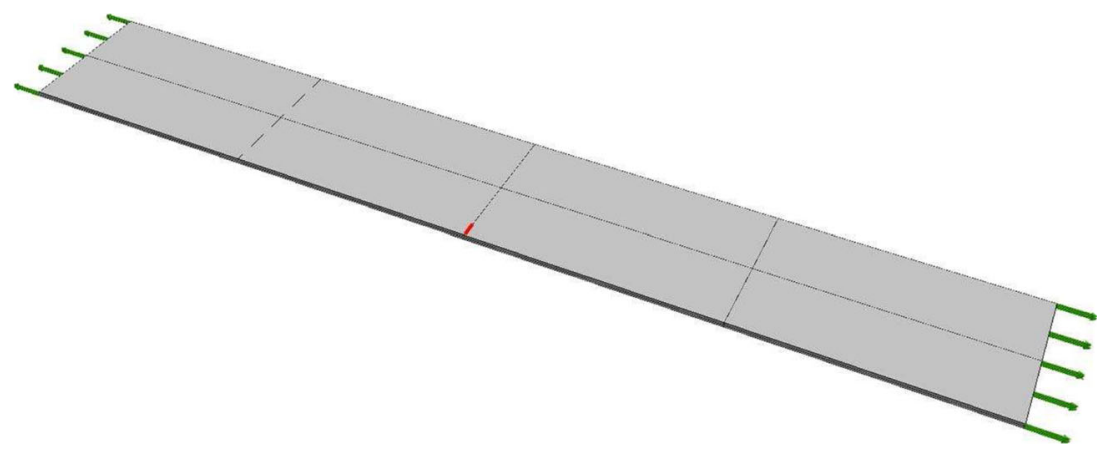




\subsection{Symmetric test case without bending action}

In order to retain a pure tearing state of the crack after adding repair patches for the specimen shown in Fig. 3, we use symmetry, which means that two identical patches are added on each side of the cracked specimen. A second symmetry plane through the crack is also used. The length of the specimen, in the direction of the distributed force, shown as green arrows in Fig. 3, is $650 \mathrm{~mm}$, and the width is $120 \mathrm{~mm}$. The thickness of the plate is $3 \mathrm{~mm}$ and the length of the crack, shown as a red color line in the figure, is $10 \mathrm{~mm}$. The design domain for topology optimization, i.e., the volume that, due to the two symmetry planes, could be occupied by half of one of the final optimized repair patches, is of size $80 \times 60 \times 25 \mathrm{~mm}$. In Fig. 4, where one of the symmetries are retained, optimal repair patches are shown in blue. The problem has been solved for different mesh refinements and for both objective functions discussed in Section 3. The volume of the optimal structure, i.e., the constant $V$ of problem $(\mathbb{P})$, is chosen as $1 / 5$ times the volume of the design domain. All used meshes consist of 27node brick elements. The results presented here are for the problem of minimizing crack energy release rate, i.e., using $f_{g}$ as objective. Results for classical stiffness maximization are presented for the more advanced test specimen in the next subsection. However, it should be noted that a repair patch that is optimal for such an objective will mostly tend to reduce the global lengthening of test specimen, instead of reducing crack opening, and, therefore, material tends to be placed away from the crack.

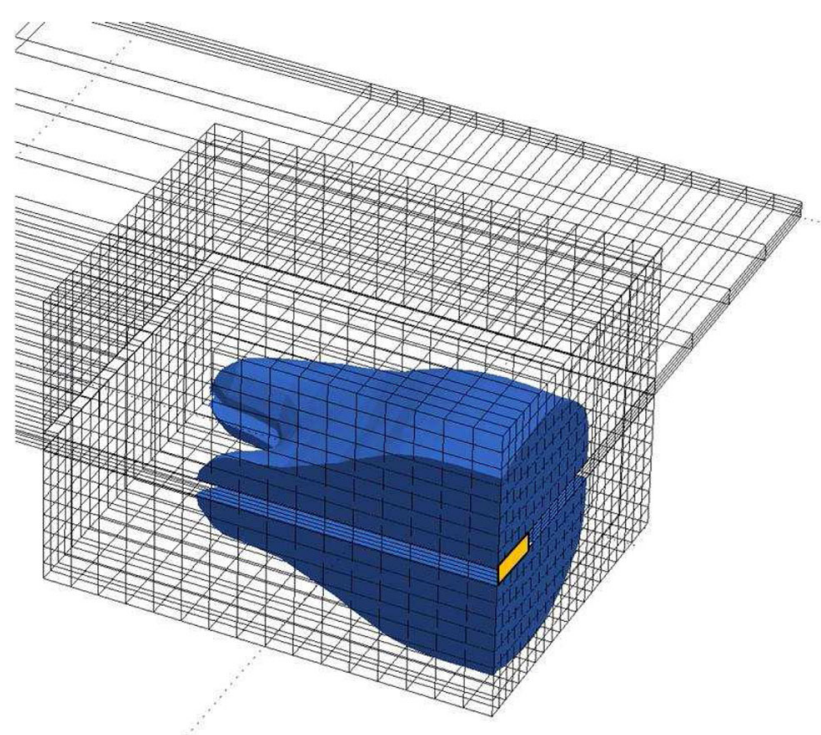

Fig. 4 Repair patches in blue obtained by minimizing energy release rate. The crack surface in the symmetry plane is shown in yellow. The picture also shows part of the finite element mesh and the design domain
In Fig. 5, the variation of optimal $G_{i}$ through the plate thickness for three different mesh refinements are shown. Note that since the average value for all three cases stays essentially the same, the behavior of the optimization problem, and, thereby, optimal repair patches are not much effected by such mesh refinements. Note also that the dropin value at the plate surfaces is in agreement with what is found in other studies (Raju et al. 1988). In fact, at such a free surface the intensity of stress singularities may vary with material constants and geometry, and while the concept of strain energy release rate stays well defined this is not so for stress intensity factors (Kuna 2013).

We have also investigated the effect of mesh refinement in the plane perpendicular to the thickness direction. Figure 6 shows the variation of average crack energy release rate $G_{a}=f_{g} / n_{c}$ with number of design elements, keeping fixed 8 elements through the thickness. This variation is small, and the shape of the optimal repair patch stays nearly unchanged. For comparison we have also calculated the average energy release rate for a flat repair patch of the same volume as the optimized ones. The dimensions are $160 \times$ $60 \times 5 \mathrm{~mm}$. The average crack energy release rate for this patch is $55 \%$ higher than that for the optimal one.

The optimal repair patch in Fig. 4 is obtained for 4 elements through the thickness, i.e., $n_{c}=5$, and the finite element mesh consists of 7680 elements, where 5120 of these make up the design domain. The value of an average crack energy release rate when adding the repair patches is only $0.14 \%$ of the value without these patches. The result in Fig. 4 was obtained after 41 iterations. Convergence behavior of the algorithm is discussed in the next subsection.

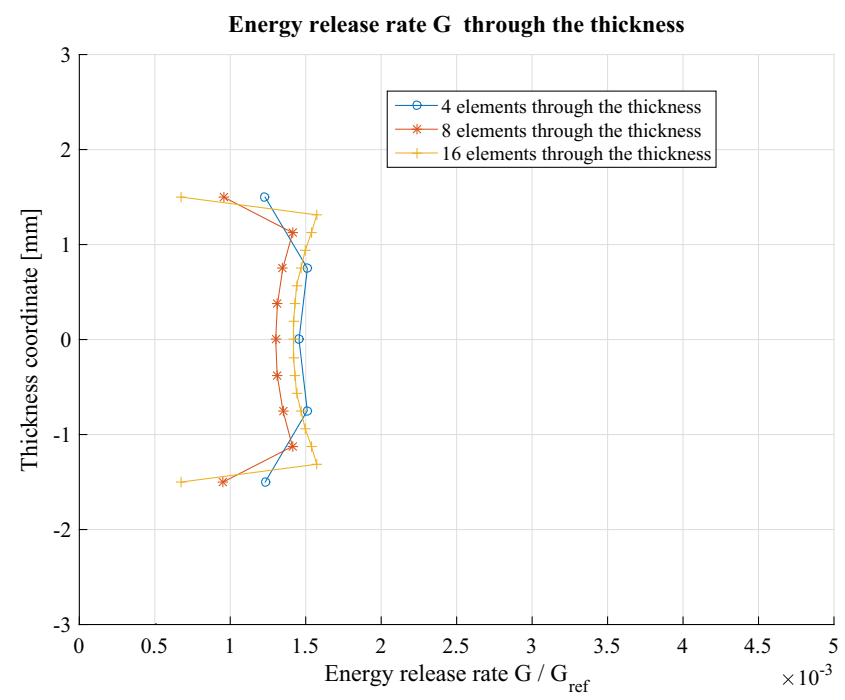

Fig. 5 Through the thickness variation of optimal energy release rate. $G_{\text {ref }}$ is the average strain energy release rate without repair patches added 
Fig. 6 Effect of mesh refinement perpendicular to the thickness direction. Variation of average crack energy release rate $G_{a} . G_{\text {ref }}$ is the average strain energy release rate without repair patches added

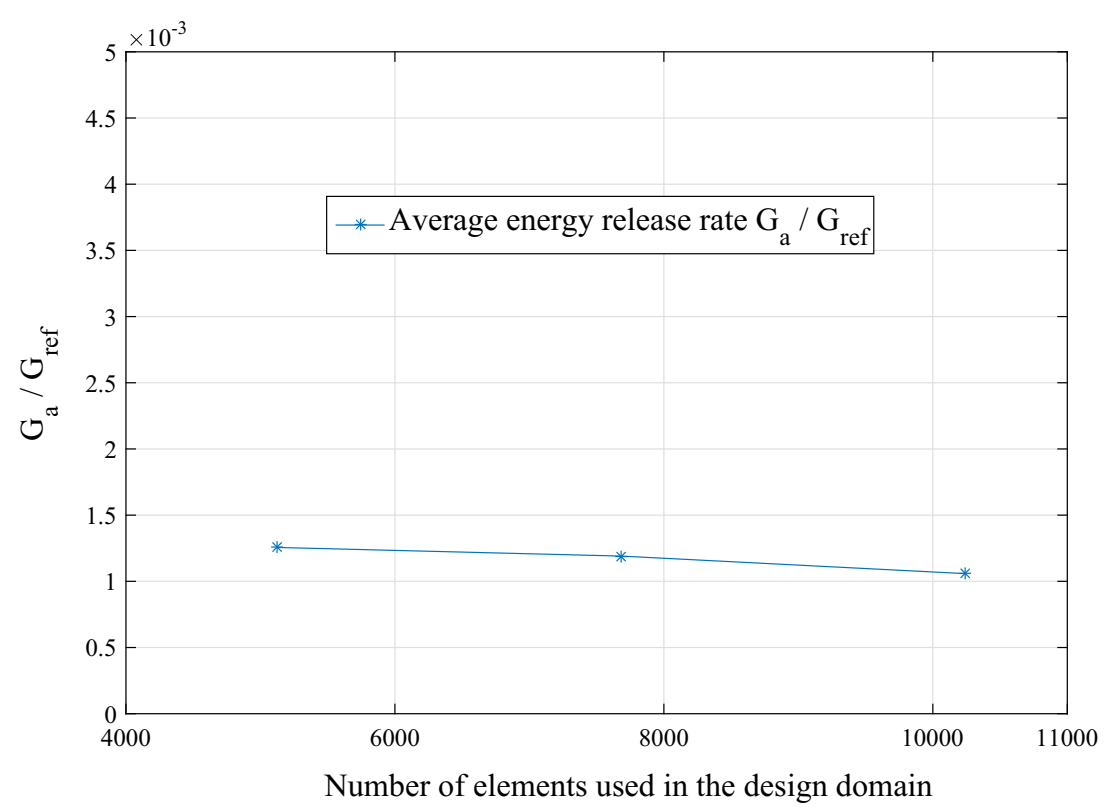

The images of the repair patches in Fig. 4, and also the corresponding patches in Figs. 8 and 9, are produced by first calculating nodal values for the design field by averaging from intersecting element values. The finite element interpolation is then used to produce a continuous design field. From this field the image is defined as the level set having values above 0.5 .

\subsection{Specimen relevant for fuselage frame}

A more advanced test specimen than that of the previous subsection is shown in Fig. 7. Although having a somewhat simple geometry, it represents flanges that are members of a principal load carrying structure in the lower section of fuselage frames. The thickness and the width of the test piece is representative for fighter airframes. It is also representative for the physical and typical limitation of the area to be reinforced with the repair patch. In Fig. 7, the through thickness crack, of length $10 \mathrm{~mm}$, is shown as a red color line and the equally distributed force as green arrows. The length of the specimen, in the direction of the force, is $420 \mathrm{~mm}$, the width of the cracked part is 120 $\mathrm{mm}$ and the height of the flange is $70 \mathrm{~mm}$. The thickness of all plates are $3 \mathrm{~mm}$. The design domain for topology optimization, i.e., the volume that could be occupied by the final optimized repair patch, is of size $50 \times 50 \times$ $15 \mathrm{~mm}$ and can be seen in Figs. 8 and 9, where optimal repair patches are shown in blue. Using symmetry, half of the structure is discretized and used in the calculations. The design domain is discretized using 19200 equally sized cubic 27-node Lagrangian elements of side length $1.25 \mathrm{~mm}$, each one connected to a design variable. The full model consists of 85618 -node wedge elements and 29184 27-node brick elements. There are 5 nodes defining the crack front, i.e., $n_{c}=5$. Also in this example the volume of the optimal repair patch is $1 / 5$ times the volume of the design domain.

Figure 8 shows the optimal repair patch using compliance $f_{c}$ as objective while Fig. 9 shows the optimal repair patch using crack energy release rate $f_{g}$. It is clear that very different optimal forms of the patches are obtained, although in both cases the force flow is directed away from the crack by the mending. However, optimizing crack energy release rate results in more material around the crack that reduces crack opening, and also in a thicker patch that

Fig. 7 A more realistic test specimen. The crack is shown as a read color line and the distributed force by green arrows

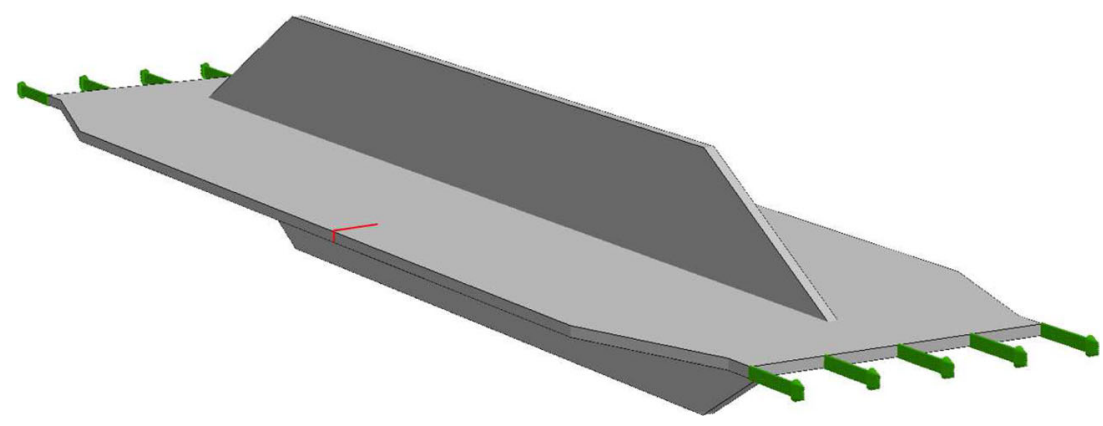




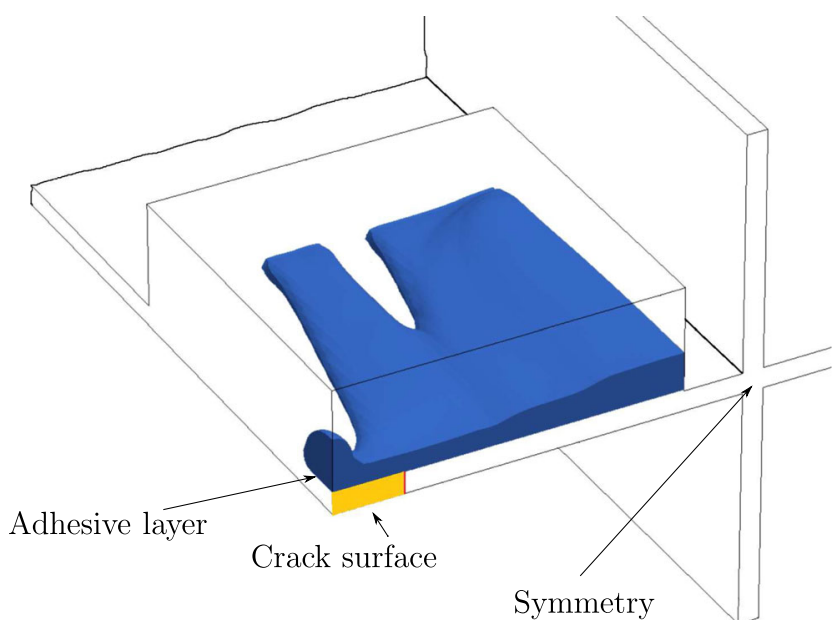

Fig. 8 By utilizing symmetry half of the structure is discretized and used in the calculations. The repair patch obtained by minimizing compliance is shown in blue

reduces bending deformation and gives a more uniform $G_{i}$ along the crack front; the variation of $G_{i}$ through the thickness of the sheet is such that is approximately 50\% smaller close to the adhesive surface, i.e., close to the repair patch, than close to the lower free surface.

Average crack energy release rates $G_{a}=f_{g} / n_{c}$, are compared for four different cases, where we take the value for a case without repair patch as reference value. If a flat repair patch, of the same volume as the optimized patches, is used, the average crack energy release rates is $18 \%$ of this reference value; for a patch optimized in stiffness as in

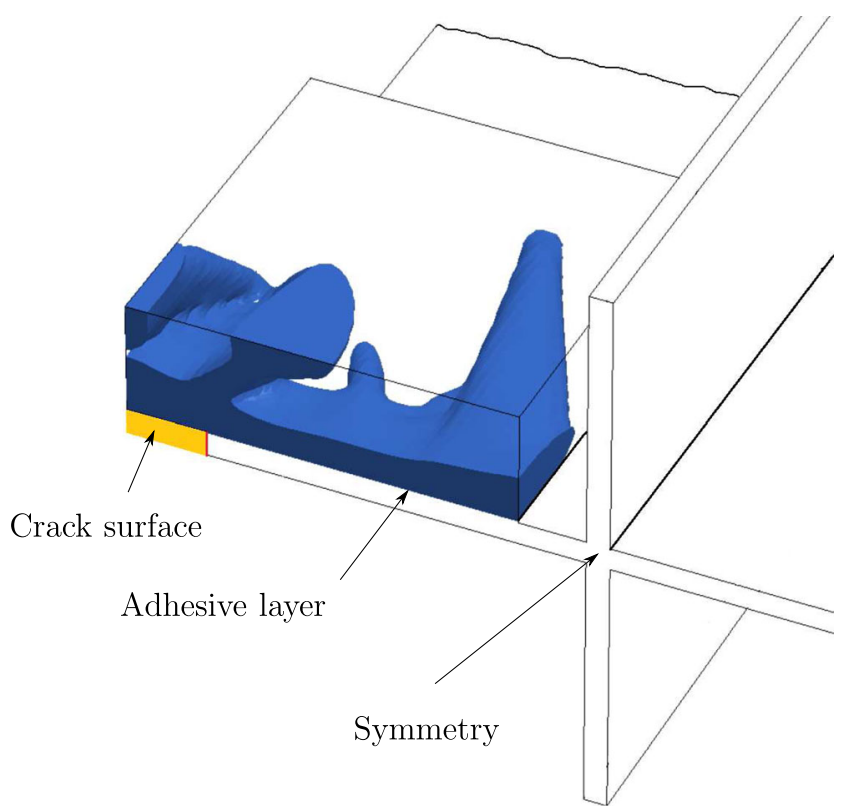

Fig. 9 The repair patch obtained by minimizing energy release rate
Fig. 8 , it is $19 \%$ of the reference value, and, finally, when $f_{g}$ is used as objective function it is $13 \%$ of the reference value.

Concerning convergence of the optimality criteria algorithm, since the number of iterations to convergence turns out to be sensitive to a prescribed tolerance, a fixed number of iterations was used. The patch in Fig. 9 was obtained after 100 iterations, which was well above a number where visual changes of the result could be detected. For the patch in Fig. 8, however, a very slow variation of the topology, while the objective function value was almost unchanged, was observed: at 100 iterations the patch consisted of two parts, while at 200 iterations the gap between these parts closed and convergence to the pictured geometry was obtained. The damping coefficient $\eta$, c.f., (9), of these calculations was 0.3. In Fig. 10 convergence histories for three different damping coefficients are compared. For $\eta=0.5$ a certain waviness is observed in the convergence curve, which levels out for lower damping coefficients. Note that monotone convergence is not obtained in any of the curves. This may be a combination of two facts: firstly, the filtering of sensitivities means that a descent direction is not guaranteed, and, secondly, no line search is included in the algorithm. It should also be understood that the low objective function values in Fig. 10, obtained after say 15 iterations, do not correspond to $0-1$ solutions, but rather contain gray regions.

In a continuation of this work experiments are to be conducted with specimens according to Fig. 7 and with repair patches resembling those of Figs. 8 and 9, as well as other shapes. Crack propagation rates will be measured

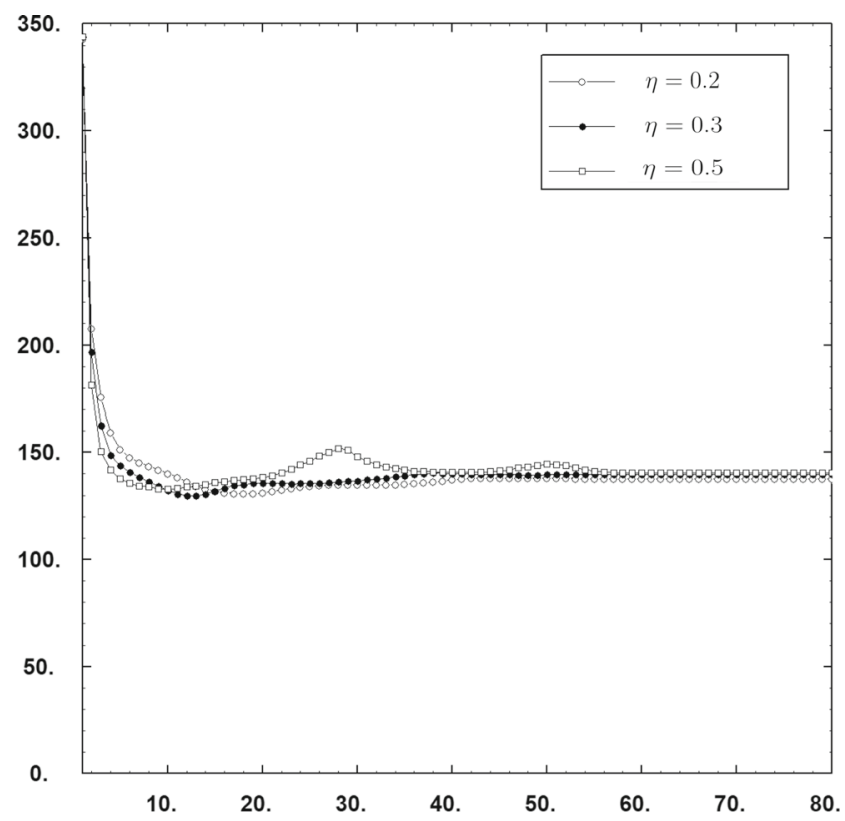

Fig. 10 Convergence history for three different damping coefficients. Number of optimality criteria updates on the horizontal axes and averaged $K_{I}$ factor, i.e., $\sqrt{E f_{g} / n_{c}}$, in units $N / m^{3 / 2}$, on the vertical axes. These values are for a total load of $1 \mathrm{~N}$ 
under constant amplitude cyclic loading both without repairs and with repair patches. The expected retardation of crack growth due to the bridging function of the repair is expected to provide evidence of weather use of optimized repair patches is preferable compared to other conventional repair solutions. More geometrically complex specimens will be tested if the results from the on-going tests are promising.

\section{Summary and conclusions}

This paper presents a novel topology optimization problem where the crack energy release rate is used as objective function. A method based on such a problem formulation enables designing a geometry that mitigate the risk for further crack growth. In particular, we design the geometry of repair patches that should be used by adhesively fixing them to a cracked structure. The application that drives the need for such a method is the possibility to repair cracks in complicated fuselage structures where replacement of structural parts may be impossible. The design method is a development of the standard SIMP method for compliance optimization and inherits its stability and simplicity. The example shows that the introduction of the designed repair patch reduces the value of the crack energy release rate substantially.

Two different objective functions are used and compared. The classical stiffness objective reduces global displacements (in the sense of $\boldsymbol{F}^{T} \boldsymbol{u}$ ) but may not be effective for reducing the risk for further crack growth, while direct use of energy release rate as objective will secure this. Intuitively, the energy release rate objective uses the available material in two ways: directly, by placing it close to the crack tip to reduce its opening and, indirectly, by redirecting the force flow away from the crack tip. Both effects are seen in the optimal repair patch of Fig. 9.

Acknowledgments The research leading to these results has received funding from VINNOVA (Swedens Innovation Agency) under program nr. 2015-06057.

Open Access This article is distributed under the terms of the Creative Commons Attribution 4.0 International License (http:// creativecommons.org/licenses/by/4.0/), which permits unrestricted use, distribution, and reproduction in any medium, provided you give appropriate credit to the original author(s) and the source, provide a link to the Creative Commons license, and indicate if changes were made.

Publisher's Note Springer Nature remains neutral with regard to jurisdictional claims in published maps and institutional affiliations.

\section{References}

Anderson TL (2017) Fracture mechanics: fundamentals and application. CRC Press, Boca Raton

Bendsøe M, Sigmund O (2002) Topology optimization, theory, methods and applications. Springer, Berlin

Brockman RA (1987) Geometric sensitivity analysis with isoparametric finite elements. Commun Appl Numer Methods 3(6):495-499

Christensen PW, Klarbring A (2009) An introduction to structural optimization. Springer, Berlin

Giner E, Fuenmayor FJ, Besa AJ, Tur M (2002) An implementation of the stiffness derivative method as discrete analytical sensitivity analysis and its application to mixed mode in LEFM. Eng Fract Mech 69:2051-2071

Hellen TK (1975) On the method of virtual crack extensions. Int J Numer Methods Eng 9(1):187-207

Holmberg E, Torstenfelt B, Klarbring A (2013) Stress constrained topology optimization. Structural and Multidisciplinary Optimization 48(1):33-47

Johansson J (1996) Crack growth simulations in Trinitas, Master thesis at Linköping University, LiTH-IKP-Ex-1354

Klarbring A, Strömberg N (2012) A note on the min-max formulation of stiffness optimization including non-zero prescribed displacements. Struct Multidiscip Optim 45:147-149

Kuna M (2013) Finite elements in fracture mechanics. Springer, Berlin

Nguyen QS (2000) Stability and nonlinear solid mechanics. Wiley, New York

Parks DM (1974) A stiffness derivative finite element technique for determination of crack tip stress intensity factors. Int J Fract 10(4):487-502

Raju IS, Hivakumar KN, Rews JH (1988) Three-dimensional elastic analysis of a composite double cantilever beam specimen. AIAA J 26(12):1493-1498

Rozvany GIN, Zhou M, Birker T (1992) Generalized shape optimization without homogenization. Struct Optim 4:250-254

Sigmund O (1994) Design of material structures using topology optimization, Ph.D. Thesis, Technical University of Denmark

Sigmund O, Petersson J (1998) Numerical instabilities in topology optimization: a survey on procedures dealing with checkerboards, mesh-dependencies and local minima. Struct Optim 16:68-75

The TRINITAS Project (2017). http://www.solid.iei.liu.se/Offered services/Trinitas/

Waisman H (2010) An analytical stiffness derivative extended finite element technique for extraction of crack tip Strain Energy Release Rates. Eng Fract Mech 77(16):3204-3215 